\title{
ASYMPTOTIC BEHAVIOR OF SINGULAR AND ENTROPY NUMBERS FOR SOME RIEMANN-LIOUVILLE TYPE OPERATORS
}

\author{
A. MESKHI
}

\begin{abstract}
The asymptotic behavior of the singular and entropy numbers is established for the Erdelyi-Köber and Hadamard integral operators (see, e.g., [15]) acting in weighted $L^{2}$ spaces. In some cases singular value decompositions are obtained as well for these integral transforms.
\end{abstract}

2000 Mathematics Subject Classification: 47B06, 47B34, 47H50.

Key words and phrases: Singular numbers, entropy numbers, RiemannLiouville operators.

In this paper, we investigate the asymptotic behavior of singular and entropy numbers for the following integral operators:

$$
I_{\alpha, \sigma} f(x)=\frac{1}{\Gamma(\alpha)} \int_{0}^{x}\left(x^{\sigma}-y^{\sigma}\right)^{\alpha-1} f(y) d y, \quad x>0, \quad \alpha>0, \quad \sigma>0,
$$

(Erdelyi-Köber operator) and

$$
H_{\alpha} f(x)=\frac{1}{\Gamma(\alpha)} \int_{1}^{x}\left(\ln \frac{x}{y}\right)^{\alpha-1} f(y) d y, \quad x>1, \quad \alpha>0,
$$

(Hadamard operator) in some weighted $L^{2}$ spaces. We get singular value decompositions for these integral transforms.

Analogous problems for the Riemann-Liouville operator

$$
R_{\alpha} f(x)=\frac{1}{\Gamma(\alpha)} \int_{0}^{x}(x-y)^{\alpha-1} f(y) d y, \quad \alpha>0,
$$

were studied in [1]-[6]. We refer also to [7]-[8], where some powerful tools were developed for establishing the asymptotics of singular numbers of certain pseudo-differential operators (see also [9] for some properties of singular numbers for the weighted Riemann-Liouville operator $R_{\alpha, v} f(x) \equiv v(x) R_{\alpha} f(x)$, where $\alpha>1 / 2$ ).

Two-sided estimates of singular (approximation) numbers for the weighted Hardy operator $\mathcal{H}_{v, w} f(x)=v(x) \int_{0}^{x} f(y) w(y) d y$ were given in [10]-[12] (for some related topics concerning the weighted Volterra integral operators see [13], [14]). 
Note that some mapping properties of the operators $I_{\alpha, \sigma}$ and $H_{\alpha}$ were established in [15].

Let $A$ and $B$ be infinite-dimensional Hilbert spaces. It is known that if $K: A \rightarrow B$ is an injective compact linear operator, then there exist:

(a) an orthonormal basis $\left\{u_{j}\right\}_{Z_{+}}$in $A$;

(b) an orthonormal basis $\left\{v_{j}\right\}_{Z_{+}}$in $B$;

(c) a nonincreasing sequence $\left\{s_{j}(K)\right\}_{Z_{+}}$of positive numbers with limit 0 as $j \rightarrow+\infty$ such that

$$
K u_{j}=s_{j}(K) v_{j}, \quad j \in Z_{+} .
$$

The numbers $s_{j}(K)$ are known as singular numbers or $s$-numbers of the operator $K$, the system $\left\{s_{j}(K), u_{j}, v_{j}\right\}_{j \in Z_{+}}$is called a singular system of $K$. For the operator $K$ the singular value decomposition

$$
K f=\sum_{j=0}^{\infty} s_{j}(K)\left(f, u_{j}\right)_{A} v_{j}, \quad f \in A,
$$

is valid.

Let $w$ be a measurable a.e. positive function on $\Omega \subset R_{+}$. We denote by $L_{w}^{2}(\Omega)$ the class of all measurable functions $f: \Omega \rightarrow R_{+}$for which

$$
\|f\|_{L_{w}^{2}(\Omega)}=\left(\int_{\Omega}|f(x)|^{2} w(x) d x\right)^{1 / 2}<\infty .
$$

In the sequel by writing $a_{n} \approx b_{n}$ for sequences of positive numbers $a_{n}$ and $b_{n}$ we mean that there exist positive constants $c_{1}$ and $c_{2}$ such that $c_{1} \leq a_{n} / b_{n} \leq c_{2}$ for all $n \in \mathbb{N}$.

The following result is well-known (see [5]):

Theorem A. Let $\alpha>0, \beta>-1, \varphi(t)=t^{-\beta} e^{-t}, \psi(t)=t^{-(\alpha+\beta)} e^{-t}$. Then the singular system $\left\{s_{j}\left(R_{\alpha}\right), u_{j}, v_{j}\right\}_{j \in Z_{+}}$of the operator $R_{\alpha}: L_{\varphi}^{2}\left(R_{+}\right) \rightarrow L_{\psi}^{2}\left(R_{+}\right)$ is given by

$$
\begin{aligned}
s_{n}\left(R_{\alpha}\right) & =\left(\frac{\Gamma(n+\beta+1)}{\Gamma(n+\alpha+\beta+1)}\right)^{1 / 2}, \\
u_{n}(t) & =\left(\frac{n !}{\Gamma(n+\beta+1)}\right)^{1 / 2} t^{\beta} L_{n}^{(\beta)}(t), \\
v_{n}(t) & =\left(\frac{n !}{\Gamma(n+\alpha+\beta+1)}\right)^{1 / 2} t^{\alpha+\beta} L_{n}^{(\alpha+\beta)}(t),
\end{aligned}
$$

and $s_{n}\left(R_{\alpha}\right) / n^{-\alpha / 2} \rightarrow 1$ as $n \rightarrow \infty$, where $L_{n}^{(\gamma)}$ is the Laguerre polynomial:

$$
L_{n}^{(\gamma)}(x)=\sum_{k=0}^{n}(-1)^{k}\left(\begin{array}{l}
n+\gamma \\
n-k
\end{array}\right) \frac{x^{k}}{k !}, \quad \gamma>-1, \quad n \in Z_{+} .
$$


Theorem B ([4]). Let $\alpha>0, \lambda>\alpha-1 / 2, \lambda \neq 0$. Then the operator $R_{\alpha}: L_{\varphi}^{2}\left(R_{+}\right) \rightarrow L_{\psi}^{2}\left(R_{+}\right)$, where $\varphi(x)=x^{1 / 2-\lambda}(1+x)^{2 \alpha}, \psi(x)=x^{1 / 2-\lambda-\alpha}$, has the following singular system:

$$
\begin{aligned}
s_{n}\left(R_{\alpha}\right) & =\left(\frac{\Gamma(n+\lambda-\alpha+1 / 2)}{\Gamma(n+\lambda+\alpha+1 / 2)}\right)^{1 / 2}, \\
u_{n}(t) & =2^{\lambda} a_{n} t^{\lambda-1 / 2}(1+t)^{-\lambda-\alpha-1 / 2} C_{n}^{\lambda}\left(\frac{1-t}{1+t}\right), \\
v_{n}(t) & =2^{\lambda} b_{n} t^{\lambda+\alpha-1 / 2}(1+t)^{-\lambda-\alpha-3 / 2} P_{n}^{(\lambda-\alpha-1 / 2, \lambda+\alpha-1 / 2)}\left(\frac{1-t}{1+t}\right),
\end{aligned}
$$

where

$$
\begin{aligned}
a_{n} & =\left(\frac{2^{2 \lambda-1}(n+\lambda) n !}{\pi \Gamma(n+2 \lambda)}\right)^{1 / 2} \Gamma(\lambda) \\
b_{n} & =\left(\frac{2^{1-2 \lambda}(n+\lambda) n ! \Gamma(n+2 \lambda)}{\Gamma(n+\lambda-\alpha+1 / 2) \Gamma(n+\lambda+\alpha+1 / 2)}\right)^{1 / 2}
\end{aligned}
$$

$C_{n}^{\lambda}(t)$ is the Gegenbauer polynomial

$$
C_{n}^{\lambda}(t)=\frac{1}{\Gamma(\alpha)} \sum_{j=0}^{[n / 2]}(-1)^{j} \frac{\Gamma(\alpha+n-j)}{j !(n-2 j) !}(2 t)^{n-2 j},
$$

and $P_{m}^{(\alpha, \beta)}$ is the Jacobi polynomial

$$
P_{n}^{(\alpha, \beta)}(t)=2^{-n} \sum_{m=0}^{n}\left(\begin{array}{c}
n+\alpha \\
m
\end{array}\right)\left(\begin{array}{c}
n+\beta \\
n-m
\end{array}\right)(t-1)^{n-m}(t+1)^{m}, \quad n \in Z_{+} .
$$

Moreover, $\lim _{n \rightarrow \infty} s_{n}\left(R_{\alpha}\right) / n^{-\alpha}=1$.

Theorem $\mathbf{C}([6])$. The singular values of the operator $R_{\alpha}: L^{2}(0,1) \rightarrow$ $L_{x^{-\gamma}}^{2}(0,1)$ have the following asymptotics:

$$
s_{n}\left(R_{\alpha}\right) \approx n^{-\alpha}, \quad 0 \leq \gamma<\alpha
$$

When $\gamma=0$, the upper estimate in the previous statement was derived in [1], [2], while the lower estimate was given in [2].

The following lemma follows immediately:

Lemma 1. Let $\varphi, \psi, v$ and $w$ be measurable a.e. positive functions on $\Omega \subseteq$ $R_{+}$. Then the operator $A$ is compact from $L_{\varphi}^{2}(\Omega)$ to $L_{\psi}^{2}(\Omega)$ if and only if the operator $A_{1} f(x)=v^{1 / 2}(x) A\left(f w^{-1 / 2}\right)(x)$ is compact from $L_{\varphi w^{-1}}^{2}(\Omega)$ to $L_{\psi v^{-1}}^{2}(\Omega)$.

Taking into account the definition of the singular system of the operator, we easily derive the next statement.

Lemma 2. Let $v$ and $w$ be a.e. positive measurable functions on $\Omega \subseteq R_{+}$. A system $\left\{s_{j}(A), u_{j}, v_{j}\right\}_{j \in Z_{+}}$is a singular system for the operator $A: L_{\varphi}^{2}(\Omega) \rightarrow$ $L_{\psi}^{2}(\Omega)$ if and only if the operator $A_{1}: L_{\varphi w^{-1}}^{2}(\Omega) \rightarrow L_{\psi v^{-1}}^{2}(\Omega)$ has the singular 
system $\left\{s_{j}\left(A_{1}\right), w^{1 / 2} u_{j}, v^{1 / 2} v_{j}\right\}_{j \in Z_{+}}$, where $A_{1} f(x)=v^{1 / 2}(x) A\left(f w^{-1 / 2}\right)(x)$ and $s_{j}\left(A_{1}\right)=s_{j}(A)$.

Let $\mathcal{I}_{\alpha, \sigma} f(x)=I_{\alpha, \sigma}(f \rho)(x)$, where $\rho(y)=y^{\sigma-1}, \alpha>0, \sigma>0$ and $x>0$.

From the definition of compactness we easily deduce

Lemma 3. Let $\alpha>0, \sigma>0$ and let $\Omega=(0,1)$ or $\Omega=(0, \infty)$. Assume that $v$ and $w$ are measurable a.e. positive functions on $\Omega$. Then the operator $\mathcal{I}_{\alpha, \sigma}$ is compact from $L_{w}^{2}(\Omega)$ to $L_{v}^{2}(\Omega)$ if and only if $R_{\alpha}$ is compact from $L_{W}^{2}(\Omega)$ to $L_{V}^{2}(\Omega)$, where $W(x)=w\left(x^{1 / \sigma}\right) x^{1 / \sigma-1}, V(x)=v\left(x^{1 / \sigma}\right) x^{1 / \sigma-1}$.

Now we prove the following statement:

Lemma 4. Let $\alpha>0, \sigma>0$ and let $v$ and $w$ be measurable a.e. positive functions on $\Omega$, where $\Omega=(0, \infty)$ or $\Omega=(0,1)$. Then for the singular system $\left\{s_{j}\left(\mathcal{I}_{\alpha, \sigma}\right\}, \bar{u}_{j}, \bar{v}_{j}\right\}_{j \in Z_{+}}$of the operator $\mathcal{I}_{\alpha, \sigma}: L_{w}^{2}(\Omega) \rightarrow L_{v}^{2}(\Omega)$ we have $s_{j}\left(\mathcal{I}_{\alpha, \sigma}\right)=$ $\sigma^{-1} s_{j}\left(R_{\alpha}\right), \bar{u}_{j}(x)=\sigma^{1 / 2} u_{j}\left(x^{\sigma}\right), \bar{v}_{j}(x)=\sigma^{1 / 2} v_{j}\left(x^{\sigma}\right)$, where $\left\{s_{j}\left(R_{\alpha}\right), u_{j}, v_{j}\right\}_{j \in Z_{+}}$ is a singular system for the operator $R_{\alpha}: L_{W}^{2}(0, \infty) \rightarrow L_{V}^{2}(0, \infty)$, with $W(x)=$ $w\left(x^{1 / \sigma}\right) x^{1 / \sigma-1}$ and $V(x)=v\left(x^{1 / \sigma}\right) x^{1 / \sigma-1}$.

Proof. Let $\Omega=(0, \infty)$. Using the change of variable $y=t^{1 / \sigma}$, we have

$$
\begin{gathered}
\left(\mathcal{I}_{\alpha, \sigma} \bar{u}_{j}\right)(x)=\frac{1}{\Gamma(\alpha)} \int_{0}^{x}\left(x^{\sigma}-y^{\sigma}\right)^{\alpha-1} y^{\sigma-1} \bar{u}_{j}(y) d y \\
=\frac{\sigma^{1 / 2}}{\Gamma(\alpha)} \int_{0}^{x}\left(x^{\sigma}-y^{\sigma}\right)^{\alpha-1} u_{j}\left(y^{\sigma}\right) y^{\sigma-1} d y=\frac{\sigma^{-1 / 2}}{\Gamma(\alpha)} \int_{0}^{x^{\sigma}}\left(x^{\sigma}-t\right)^{\alpha-1} u_{j}(t) d t \\
=\sigma^{-1 / 2}\left(R_{\alpha} u_{j}\right)\left(x^{\sigma}\right)=s_{j}\left(R_{\alpha}\right) \sigma^{-1 / 2} v_{j}\left(x^{\sigma}\right)=\sigma^{-1} s_{j}\left(R_{\alpha}\right) \bar{v}_{j}(x) .
\end{gathered}
$$

Further, the change of variable yields

$$
\begin{gathered}
\int_{0}^{\infty} \bar{v}_{j}(x) \bar{v}_{i}(x) v(x) d x=\sigma \int_{0}^{\infty} v_{j}\left(x^{\sigma}\right) v_{i}\left(x^{\sigma}\right) V\left(x^{\sigma}\right) x^{\sigma-1} d x \\
=\int_{0}^{\infty} v_{j}(x) v_{i}(x) V(x) d x=\delta_{i j},
\end{gathered}
$$

where $\delta_{i j}$ denotes Kronecker's symbol.

Analogously, we have

$$
\int_{0}^{\infty} \bar{u}_{j}(x) \bar{u}_{i}(x) w(x) d x=\int_{0}^{\infty} u_{j}(x) u_{i}(x) W(x) d x=\delta_{i j},
$$

Hence $\left\{\bar{v}_{j}\right\}$ and $\left\{\bar{u}_{j}\right\}$ are orthonormal systems in $L_{v}^{2}\left(R_{+}\right)$and $L_{w}^{2}\left(R_{+}\right)$, respectively.

The case $\Omega=(0,1)$ follows in a similar way. 
Theorem 1. Let $\alpha>0, \sigma>0$ and $0 \leq \gamma<\alpha$. Then there exist positive constants $c_{1}$ and $c_{2}$ depending on $\alpha, \sigma$ and $\gamma$ such that for the singular numbers of the operator $I_{\alpha, \sigma}: L_{x^{1-\sigma}}^{2}(0,1) \rightarrow L_{x^{\sigma-1-\gamma \sigma}}^{2}(0,1)$ we have $s_{n}\left(I_{\alpha, \sigma}\right) \approx n^{-\alpha}$.

Proof. By Lemma 2 we have that $s_{j}\left(I_{\alpha, \sigma}\right)=s_{j}\left(\mathcal{I}_{\alpha, \sigma}\right)$, where $\mathcal{I}_{\alpha, \sigma}$ acts from $L_{x^{\sigma-1}}^{2}(0,1)$ to $L_{x^{\sigma-1-\gamma \sigma}}^{2}(0,1)$, while Lemma 4 yields $s_{j}\left(\mathcal{I}_{\alpha, \sigma}\right)=1 / \sigma s_{j}\left(R_{\alpha}\right)$, where $R_{\alpha}$ is the Riemann-Liouville operator acting from $L^{2}(0,1)$ to $L_{x^{-\gamma}}^{2}(0,1)$. Theorem $\mathrm{C}$ completes the proof.

Theorem 2. Let $\alpha>0, \sigma>0, \lambda>\alpha-1 / 2$ and $\lambda \neq 0$. Assume that $w(x)=x^{1-\sigma / 2-\sigma \lambda}\left(1+x^{\sigma}\right)^{2 \alpha}, v(x)=x^{3 \sigma / 2-\sigma \lambda-\sigma \alpha-1}$. Then the operator $I_{\alpha, \sigma}$ : $L_{w}^{2}(0, \infty) \rightarrow L_{v}^{2}(0, \infty)$ has a singular system $\left\{s_{n}\left(I_{\alpha, \sigma}\right), \bar{u}_{n}, \bar{v}_{n}\right\}_{n \in Z_{+}}$, where

$$
\begin{aligned}
s_{n}\left(I_{\alpha, \sigma}\right) & =1 / \sigma\left(\frac{\Gamma(n+\lambda-\alpha+1 / 2)}{\Gamma(n+\lambda+\alpha+1 / 2)}\right)^{1 / 2}, \\
\bar{u}_{n}(x) & =\sigma^{1 / 2} 2^{\lambda} a_{n} x^{\sigma(\lambda+1 / 2)-1}\left(1+x^{\sigma}\right)^{-\lambda-\alpha-1 / 2} C_{n}^{\lambda}\left(\frac{1-x^{\sigma}}{1+x^{\sigma}}\right), \\
\bar{v}_{n}(x) & =\sigma^{1 / 2} 2^{\lambda} b_{n} x^{\sigma(\lambda+\alpha-1 / 2)}\left(1+x^{\sigma}\right)^{-\lambda-\alpha-3 / 2} P_{n}^{(\lambda-\alpha-1 / 2, \lambda+\alpha-1 / 2)}\left(\frac{1-x^{\sigma}}{1+x^{\sigma}}\right),
\end{aligned}
$$

$C_{n}^{\lambda}(x)$ and $P_{n}^{(\alpha, \beta)}$ are Gegenbauer and Jacobi polynomials, respevetively (see Theorem B), and $a_{n}, b_{n}$ are the constants defined in Theorem B. Moreover,

$$
\lim _{n \rightarrow \infty} s_{n}\left(I_{\alpha, \sigma}\right) / n^{-\alpha}=1 / \sigma .
$$

Proof. Lemma 2 implies that the singular system $\left\{s_{m}\left(I_{\alpha, \sigma}\right), \bar{u}_{m}, \bar{v}_{m}\right\}_{m \in Z_{+}}$of the map $I_{\alpha, \sigma}: L_{w}^{2}(0, \infty) \rightarrow L_{v}^{2}(0, \infty)$ coincides with the singular system $\left\{s_{m}\left(\mathcal{I}_{\alpha, \sigma}\right), \widetilde{u}_{m}\right.$, $\left.\widetilde{v}_{m}\right\}_{m \in Z_{+}}$of the map $\mathcal{I}_{\alpha, \sigma}: L_{W}^{2}(0, \infty) \rightarrow L_{V}^{2}(0, \infty)$, where $W(x)=w(x) x^{2(\sigma-1)}$, $V(x)=v(x), \widetilde{u}_{m}(x)=x^{1-\sigma} u_{m}(x), \widetilde{v}_{m}(x)=\bar{v}_{m}(x)$. Further, by Lemma 4 we have that the operator $R_{\alpha}: L_{\varphi}^{2}(0, \infty) \rightarrow L_{\psi}^{2}(0, \infty)\left(\varphi(x)=x^{1 / 2-\lambda}(1+x)^{2 \alpha}\right.$, $\psi(x)=x^{1 / 2-\lambda-\alpha}$ ) has a singular system $\left\{s_{m}\left(R_{\alpha}\right), u_{m}, v_{m}\right\}_{m \in Z_{+}}$, where

$$
s_{m}\left(R_{\alpha}\right)=\sigma s_{m}\left(\mathcal{I}_{\alpha \sigma}\right) \approx m^{-\alpha}, \quad \bar{u}_{m}(x)=\sigma^{1 / 2} x^{\sigma-1} u_{m}\left(x^{\sigma}\right), \quad \bar{v}_{m}(x)=\sigma^{1 / 2} v_{m}\left(x^{\sigma}\right) .
$$

Analogously, we have

Theorem 3. Let $\alpha>0, \sigma>0, \beta>-1, w(y)=y^{-\sigma \beta-\sigma+1} e^{-y^{\sigma}}$ and $v(y)=$ $y^{-\sigma(\alpha+\beta)+\sigma-1} e^{-y^{\sigma}}$. Then the operator $I_{\alpha, \sigma}: L_{w}^{2}(0, \infty) \rightarrow L_{v}^{2}(0, \infty)$ has a singular system $\left\{s_{m}\left(I_{\alpha, \sigma}\right), \bar{u}_{m}, \bar{v}_{m}\right\}_{m \in Z_{+}}$defined by

$$
\begin{aligned}
s_{n}\left(I_{\alpha, \sigma}\right) & =1 / \sigma\left(\frac{\Gamma(n+\beta+1)}{\Gamma(n+\alpha+\beta+1)}\right)^{1 / 2}, \\
\bar{u}_{n}(x) & =\sigma^{1 / 2} x^{\sigma-1+\sigma \beta}\left(\frac{n !}{\Gamma(n+\beta+1)}\right)^{1 / 2} L_{n}^{(\beta)}\left(x^{\sigma}\right), \\
\bar{v}_{n}(x) & =\sigma^{1 / 2}\left(\frac{n !}{\Gamma(n+\alpha+\beta+1)}\right)^{1 / 2} x^{\sigma(\alpha+\beta)} L_{n}^{(\alpha+\beta)}\left(x^{\sigma}\right),
\end{aligned}
$$


where $L_{n}^{(\gamma)}(x)$ is a Laguerre polynomial (see Theorem A). Moreover,

$$
\lim _{n \rightarrow \infty} s_{n}\left(I_{\alpha, \sigma}\right) / n^{-\alpha / 2}=1 / \sigma .
$$

Now we consider the operator of Hadamard's type $H_{\alpha}$.

The following lemma holds:

Lemma 5. Let $\alpha>0$ and $(v, w)$ be a pair of weights defined on $(1, \infty)$. Then $\left\{s_{m}\left(L_{\alpha}\right), \bar{u}_{m}, \bar{v}_{m}\right\}_{m \in Z_{+}}$is a singular system for the operator $L_{\alpha}: L_{w}^{2}(1, \infty) \rightarrow$ $L_{v}^{2}(1, \infty)$, where

$$
L_{\alpha} f(x)=\frac{1}{\Gamma(\alpha)} \int_{1}^{x}\left(\ln \frac{x}{y}\right)^{\alpha-1} f(y) \frac{d y}{y},
$$

if and only if the Riemann-Liouville operator $R_{\alpha}: L_{W}^{2}(0, \infty) \rightarrow L_{V}^{2}(0, \infty)$ has a singular system $\left\{s_{m}\left(R_{\alpha}\right), \widetilde{u}_{m}, \widetilde{v}_{m}\right\}_{m \in Z_{+}}$, where $W(x)=w\left(e^{x}\right) e^{x}, V(x)=$ $v\left(e^{x}\right) e^{x}, s_{m}\left(R_{\alpha}\right)=s_{m}\left(L_{\alpha}\right), \widetilde{u}_{m}(x)=\bar{u}_{m}\left(e^{x}\right), \widetilde{v}_{m}(x)=\bar{v}_{m}\left(e^{x}\right)$.

Proof. Using the change of variable $y=e^{z}$ we have

$$
\begin{gathered}
\left(L_{\alpha} \bar{u}_{m}\right)(x)=\frac{1}{\Gamma(\alpha)} \int_{1}^{x}\left(\ln \frac{x}{y}\right)^{\alpha-1} \bar{u}_{m}(y) \frac{d y}{y} \\
=\frac{1}{\Gamma(\alpha)} \int_{0}^{\ln x}(\ln x-z)^{\alpha-1} \widetilde{u}_{m}(z) d z=\left(R_{\alpha} \widetilde{u}_{m}\right)(\ln x)=\widetilde{v}(\ln x) s_{j}\left(R_{\alpha}\right) .
\end{gathered}
$$

On the other hand,

$$
\begin{gathered}
\int_{0}^{\infty} \widetilde{u}_{i}(x) \widetilde{u}_{j}(x) W(x) d x=\int_{0}^{\infty} \bar{u}_{i}\left(e^{x}\right) \bar{u}_{j}\left(e^{x}\right) w\left(e^{x}\right) e^{x} d x=\delta_{i j}, \\
\int_{0}^{\infty} \widetilde{v}_{i}(x) \widetilde{v}_{j}(x) V(x) d x=\int_{1}^{\infty} \bar{v}_{i}(y) \bar{v}_{j}(y) v(y) d y=\delta_{i j},
\end{gathered}
$$

where $\delta_{i j}$ is Kronecker's symbol.

Lemmas 2 and 5 yield the following statements:

Theorem 4. Let $\alpha>0, \beta>-1, w(x)=\ln ^{-\beta} x, v(x)=x^{-2} \ln ^{-(\alpha+\beta)} x$. Then the operator $H_{\alpha}: L_{w}^{2}(1, \infty) \rightarrow L_{v}^{2}(1, \infty)$ has a singular system $\left\{s_{n}\left(H_{\alpha}\right), \widetilde{u}_{n}, \widetilde{v}_{n}\right\}_{n \in Z_{+}}$, where $s_{n}\left(H_{\alpha}\right)=s_{n}\left(R_{\alpha}\right)\left(s_{m}\left(R_{\alpha}\right)\right.$ is defined by $\left.(1)\right)$,

$$
\begin{aligned}
& \widetilde{u}_{n}(x)=x^{-1}\left(\frac{n !}{\Gamma(n+\beta+1)}\right)^{1 / 2} L_{n}^{(\beta)}(\ln x) \ln ^{\beta} x, \\
& \widetilde{v}_{n}(x)=\left(\frac{n !}{\Gamma(n+\alpha+\beta+1)}\right)^{1 / 2} L_{n}^{(\alpha+\beta)}(\ln x) \ln ^{\alpha+\beta} x,
\end{aligned}
$$

and $L_{n}^{(\gamma)}$ is the Laguerre polynomial. Moreover,

$$
\lim _{n \rightarrow \infty} s_{n}\left(H_{\alpha}\right) / n^{-\alpha / 2}=1 \text {. }
$$


Theorem 5. Let $\lambda>\alpha-\frac{1}{2}, \lambda \neq 0$. Then the operator $H_{\alpha}: L_{w}^{2}(1, \infty) \rightarrow L_{v}^{2}(1, \infty)$ has a singular system $\left\{s_{n}\left(H_{\alpha}\right), \widetilde{u}_{m}, \widetilde{v}_{n}\right\}_{m \in Z_{+}}$, where $v(x)=x^{-1} \ln ^{1 / 2-\lambda-\alpha} x$, $w(x)=(1+\ln x)^{2 \alpha} x \ln ^{1 / 2-\lambda} x, s_{n}\left(H_{\alpha}\right)=s_{n}\left(R_{\alpha}\right)\left(s_{n}\left(R_{\alpha}\right)\right.$ is defined by $\left.(2)\right)$,

$$
\begin{aligned}
& \widetilde{u}_{n}(x)=2^{\lambda} a_{n}(1+\ln x)^{-\lambda-\alpha-1 / 2} C_{n}^{\lambda}\left(\frac{1-\ln x}{1+\ln x}\right) x^{-1} \ln ^{\lambda-1 / 2} x, \\
& v_{n}(x)=2^{\lambda} b_{n}(1+\ln x)^{-\lambda-\alpha-3 / 2} P_{n}^{(\lambda-\alpha-1 / 2, \lambda+\alpha-1 / 2)}\left(\frac{1-\ln x}{1+\ln x}\right) \ln ^{\lambda+\alpha-1 / 2} x .
\end{aligned}
$$

Moreover,

$$
\lim _{n \rightarrow \infty} s_{n}\left(H_{\alpha}\right) / n^{-\alpha}=1 .
$$

Definition 1. Let $X$ and $Y$ be Banach spaces and let $T$ be a bounded linear map from $X$ to $Y$. Then for all $k \in N$, the $k^{t h}$ entropy number $e_{k}(T)$ of $T$ is defined by

$$
e_{k}(T)=\inf \left\{\varepsilon>0: T\left(U_{X}\right) \subset \bigcup_{j=1}^{2^{k-1}}\left(b_{i}+\varepsilon U_{Y}\right) \text { for some } b_{1}, \ldots, b_{2^{k-1}} \in Y\right\},
$$

where $U_{X}$ and $U_{Y}$ are the closed unit balls in $X$ and $Y$, respectively.

It is easy to verify that $\|T\|=e_{1}(T) \geq e_{2}(T) \geq \cdots \geq 0$.

For other properties of the entropy numbers see, e.g., [16].

It is known (see, e.g., [15]), that if $T$ is a compact linear map of a Hilbert space $X$ into a Hilbert space $Y$, then $s_{n}(T) \approx n^{-\lambda}$ if and only if $e_{n}(T) \approx n^{-\lambda}$. Hence we can get asymptotics of the entropy numbers for the operators $I_{\alpha, \sigma}$ and $H_{\alpha}$. In particular, Theorems 1, 2 and 3 yield

Proposition 1. Let $\alpha>0$ and $\sigma>0$. Then the following statements are valid:

(a) If $0 \leq \gamma<\alpha$, then the asymptotic formula

$$
e_{n}\left(I_{\alpha, \sigma}\right) \approx n^{-\alpha}
$$

holds for the operator $I_{\alpha, \sigma}: L_{x^{1-\sigma}}^{2}(0,1) \rightarrow L_{x^{\sigma-1-\gamma \sigma}}^{2}(0,1)$.

(b) Assume that $\lambda>\alpha-1 / 2$ and $\lambda \neq 0$. Then the asymptotic formula (3) is valid for the map $I_{\alpha, \sigma}: L_{w}^{2}(0, \infty) \rightarrow L_{v}^{2}(0, \infty)$, where $w(x)=x^{-\sigma / 2-\sigma \lambda+1}(1+$ $\left.x^{\sigma}\right)^{2 \alpha}$ and $v(x)=x^{3 \sigma / 2-\sigma \lambda-\sigma \alpha-1}$.

(c) For the entropy numbers $e_{n}\left(I_{\alpha, \sigma}\right)$ of the operator $I_{\alpha, \sigma}: L_{w}^{2}(0, \infty) \rightarrow$ $L_{v}^{2}(0, \infty)\left(w(y)=y^{-\sigma \beta-\sigma+1} e^{-y^{\sigma}}, v(y)=y^{-\sigma(\alpha+\beta)+\sigma-1} e^{-y^{\sigma}}, \beta>-1\right)$ we have

$$
e_{n}\left(I_{\alpha, \sigma}\right) \approx n^{-\alpha / 2}
$$

Let $T: L_{w}^{2} \rightarrow L_{v}^{2}$ be a compact linear operator. We shall denote by $n(t, T)$ the distribution function of singular values for the operator $T$, i.e.,

$$
n(t, T) \equiv \sharp\left\{k: s_{k}(T)>t\right\} .
$$


Theorem 6. Let $\alpha>1 / 2$ and $\sigma>0$. Assume that $v$ is a measurable a.e. positive function of $(0, \infty)$ satisfying the condition

$$
\sum_{k \in \mathbb{Z}}\left(\int_{2^{k / \sigma}}^{2^{(k+1) / \sigma}} v(y) y^{(2 \alpha-1) \sigma} d y\right)^{1 /(2 \alpha)}<\infty .
$$

Then for the operator $I_{\alpha, \sigma}: L_{w}^{2}\left(R_{+}\right) \rightarrow L_{v}^{2}\left(R_{+}\right)$, where $w(x)=x^{1-\sigma}$, the asymptotic formula

$$
\lim _{t \rightarrow 0} t^{1 / \alpha} n\left(t, I_{\alpha, \sigma}\right)=\frac{\sigma^{-1 / \alpha+1}}{\pi} \int_{0}^{\infty} v^{1 /(2 \alpha)}(y) y^{(1-\sigma)(1 /(2 \alpha)-1)} d y
$$

holds.

Proof. Condition (4) implies that

$$
\sum_{k \in \mathbb{Z}}\left(\int_{2^{k}}^{2^{k+1}} \bar{v}^{2}(y) y^{2 \alpha-1} d y\right)^{1 /(2 \alpha)}<\infty
$$

where $\bar{v}(x) \equiv\left[v\left(x^{1 / \sigma}\right) x^{1 / \sigma-1}\right]^{1 / 2}$. By virtue of Theorem 1 from [9] we have that for the operator $R_{\alpha, \bar{v}}: L^{2}\left(R_{+}\right) \rightarrow L^{2}\left(R_{+}\right)$, where $R_{\alpha, \bar{v}} f(x) \equiv \bar{v}(x) R_{\alpha} f(x)$, the asymptotic formula

$$
\lim _{t \rightarrow 0} t^{1 / \alpha} n\left(t, R_{\alpha, \bar{v}}\right)=\pi^{-1} \int_{R_{+}} \bar{v}^{1 / \alpha}(x) d x
$$

holds. Further, using Lemmas 1, 2 and 3 we obtain that $s_{k}\left(R_{\alpha, \bar{v}}\right)=\sigma \cdot s_{k}\left(I_{\alpha, \sigma}\right)$. Consequently,

$$
\begin{gathered}
\lim _{t \rightarrow 0} t^{1 / \alpha} n\left(t, I_{\alpha, \sigma}\right)=\sigma^{-1 / \alpha} \lim _{t \rightarrow 0} t^{1 / \alpha} n\left(t, R_{\alpha, \bar{v}}\right) \\
=\sigma^{-1 / \alpha} \frac{1}{\pi} \int_{0}^{\infty}(\bar{v}(x))^{1 / \alpha} d x=\frac{\sigma^{-1 / \alpha+1}}{\pi} \int_{0}^{\infty}(v(y))^{1 /(2 \alpha)} y^{(1-\sigma)(1 /(2 \alpha)-1)} d y .
\end{gathered}
$$

Theorem 7. Let $\alpha>1 / 2$ and $\sigma>0$. Suppose that $v$ is a measurable a.e. positive function on $(0,1)$ satisfying the condition

$$
\sum_{k \in \mathbb{Z}}\left(\int_{a_{k}}^{a_{k+1}} v(x) x^{-\sigma+2 \alpha \sigma}\left(1-x^{\sigma}\right)^{-1} d x\right)^{1 /(2 \alpha)}<\infty, \quad a_{k}=\left(2^{k} /\left(2^{k}+1\right)\right)^{1 / \sigma} .
$$

Then for the operator $I_{\alpha, \sigma}$ acting from $L_{w}^{2}(0,1)$ into $L_{v}^{2}(0,1)$, where $w(x)=$ $\left(1-x^{\sigma}\right)^{2 \alpha} x^{1-\sigma}$, we have

$$
\lim _{t \rightarrow 0} t^{1 / \alpha} n\left(t, I_{\alpha, \sigma}\right)=\frac{\sigma^{-1 / \alpha+1}}{\pi} \int_{0}^{1} v^{1 /(2 \alpha)}(x) x^{(1-\sigma)(1 /(2 \alpha)-1)}\left(1-x^{\sigma}\right)^{-1} d x .
$$


Proof. Using Lemmas 1-4 we have that $s_{n}\left(I_{\alpha, \sigma}\right)=1 / \sigma s_{n}\left(R_{\alpha}\right)$, where $R_{\alpha}$ is the Riemann-Liouville operator acting from $L_{w_{1}}^{2}(0,1)$ into $L_{v_{1}}^{2}(0,1)$, with

$$
w_{1}(x)=w\left(x^{1 / \sigma}\right) x^{1-1 / \sigma}, \quad v_{1}(x)=v\left(x^{1 / \sigma}\right) x^{1 / \sigma-1} .
$$

Further, by the change of variable $x=y /(1-y)$ we obtain that the operator $\bar{R}_{\alpha}: L_{w_{2}}^{2}\left(R_{+}\right) \rightarrow L_{v_{2}}^{2}\left(R_{+}\right)$has singular numbers $s_{n}\left(\bar{R}_{\alpha}\right)=\sigma s_{n}\left(I_{\alpha, \sigma}\right)$, where $w_{2}(x)=w_{1}(x /(x+1))(x+1)^{-2}, v_{2}(x)=v_{1}(x /(x+1))(x+1)^{-2}$ and $\bar{R}_{\alpha} f(x)=$ $\psi(x) R_{\alpha}(f \varphi)(x)$ with $\psi(x)=(x+1)^{-\alpha+1}, \varphi(x)=(x+1)^{-1-\alpha}$. Hence for the singular numbers of the Riemann-Liouville operator $R_{\alpha}: L_{w_{3}}^{2}\left(R_{+}\right) \rightarrow L_{v_{3}}^{2}\left(R_{+}\right)$ we derive $s_{n}\left(R_{\alpha}\right)=\sigma s_{n}\left(I_{\alpha, \sigma}\right)$, where $w_{3}(x)=w_{2}(x)(x+1)^{2 \alpha+2}=1$ and $v_{3}(x)=$ $v_{2}(x)(x+1)^{2-2 \alpha}$. Further, condition (6) implies (5) with $v_{3}$ instead of $v$. Thus, taking into account Theorem 1 from [9], we arrive at

$$
\begin{gathered}
\lim _{t \rightarrow 0} t^{1 / \alpha} n\left(t, I_{\alpha, \sigma}\right)=\sigma^{-1 / \alpha} \lim _{t \rightarrow 0} t^{1 / \alpha} n\left(t, R_{\alpha}\right) \\
=\sigma^{-1 / \alpha} \frac{1}{\pi} \int_{0}^{\infty} v_{4}^{1 / \alpha}(x) d x=\frac{\sigma^{-1 / \alpha+1}}{\pi} \int_{0}^{1}(v(y))^{1 /(2 \alpha)} y^{(1-\sigma)(1 /(2 \alpha)-1)}\left(1-y^{\sigma}\right)^{-1} d y .
\end{gathered}
$$

In the last equality we used the change of variable twice.

Finally, we have

Theorem 8. Let $\alpha>1 / 2$ and let $v$ be a measurable a.e. positive function on $(1, \infty)$ satisfying the condition

$$
\sum_{k \in \mathbb{Z}}\left(\int_{a_{k}}^{a_{k+1}} v(x) \ln ^{2 \alpha-1} x d x\right)^{1 /(2 \alpha)}<\infty, \quad a_{k}=e^{2^{k}} .
$$

Then for the operator $H_{\alpha}: L_{w}^{2}(1, \infty) \rightarrow L_{v}^{2}(1, \infty)$, where $w(x)=e^{x}$, the asymptotic formula

$$
\lim _{t \rightarrow 0} t^{1 / \alpha} n\left(t, H_{\alpha, \sigma}\right)=\frac{1}{\pi} \int_{1}^{\infty} v^{1 /(2 \alpha)}(x) x^{1 /(2 \alpha)-1} d y
$$

holds.

Proof. Taking into account Lemmas 2 and 5 we obtain that $s_{n}\left(R_{\alpha}\right)=s_{n}\left(H_{\alpha}\right)$, where $R_{\alpha}$ is the Riemann-Liouville operator acting from $L^{2}\left(R_{+}\right)$into $L_{v_{1}}^{2}\left(R_{+}\right)$, $v_{1}(x)=v\left(e^{x}\right) e^{x}$. By condition (7), Theorem 1 from [9] and the change of variable $x=e^{y}$ we conclude that (8) holds.

\section{ACKNOWLEDGEMENT}

The work was partially supported by Grant No. 1.7 of the Georgian Academy of Sciences. 


\section{REFERENCES}

1. V. FABER and G. M. Wing, Singular values of fractional integral operators: a unification of theorems of Hille, Tamarkin, and Chang. J. Math. Anal. Appl. 120(1986), 745-760.

2. M. R. Dostenić, Asymptotic behavior of the singular value of fractional integral operators. J. Math. Anal. Appl. 175(1993), 380-391.

3. J. Baumeister, Stable solution of inverse problems. Vieweg and Sohn, Braunschweig/Wiesbaden, 1987.

4. R. Gorenflo and S. Samko, On the dependence of asymptotics of $s$-numbers of fractional integration operators on weight functions. Preprint No. A-3/96, Freie Universität Berlin, Fachbereich Mathematik und Informatik, Serie A, Mathematik, 1996.

5. R. Gorenflo and Vu Kim Tuan, Singular value decomposition of fractional integration operators in $L_{2}-$ spaces with weights. J. Inv. Ill- Posed Problems 3(1995), No. 1, 1-9.

6. R. Gorenflo and Vu Kim Tuan, Asymptotics of singular values of fractional integration operators. Preprint No. A-3/94, Freie Universität Berlin, Fachbereich Mathematik und Informatik, Serie A, Mathematik, 1994.

7. M. Sh. Birman and M. Solomyak, Estimates for the singular numbers of integral operators. (Russian) Uspekhi Mat. Nauk 32(1997), No. 1, 17-82; English translation: Russian Math. Surveys 32(1977).

8. M. Sh. Birman and M. Solomyak, Asymptotic behavior of the spectrum differential operators with anisotropically homogeneous symbols. (Russian) Vestnik Leningrad. Univ. 13(1977), 13-21; English translation: Vestnik Leningrad Univ. Math. 10(1982).

9. J. Newmann and M. Solomyak, Two-sided estimates on singular values for a class of integral operators on the semi-axis. Integral Equations Operator Theory 20(1994), 335349.

10. D. E. Edmunds, W. D. Evans, and D. J. Harris, Approximation numbers of certain Volterra integral operators. J. London Math. Soc. 37(1988), No. 2, 471-489.

11. D. E. Edmunds, W. D. Evans, and D. J. Harris, Two-sided estimates of the approximation numbers of certain Volterra integral operators. Studia Math. 124(1997), No. 1, 59-80.

12. D. E. Edmunds, R. Kerman, and J. LAng, Remainder estimates for the approximation numbers of weighted Hardy operators acting on $L^{2}$. Research Report No: 2000-11, University of Sussex at Brighton, 2000.

13. A. Meskhi, On the measure of non-compactness and singular numbers for the Volterra integral operators. Proc. A. Razmadze Math. Inst. 123(2000), 162-165.

14. A. Meskni, On the singular numbers for some integral operators. Revista Mat. Comp. 14(2001), No. 2.

15. S. G. Samko, A. A. Kilbas, and O. I. Marichev, Integrals and derivatives. Theory and Applications. Gordon and Breach Science Publishers, London-New York, 1993.

16. D. E. Edmunds and H. Triebel, Function spaces, entropy numbers, differential operators. Cambridge University Press, Cambridge, 1996.

17. B. CARL, Inequalities of Bernstein-Jackson type and the degree of compactness of operators in Banach spaces. Ann. Inst. Fourier 35(1985), 79-118.

(Received 20.03.2001)

Author's address:

A. Razmadze Mathematical Institute, Georgian Academy of Sciences

1, M. Aleksidze St., Tbilisi 380093, Georgia

E-mail: meskhi@rmi.acnet.ge 